\title{
Circulating osteocalcin level is associated with improved glucose tolerance, insulin secretion and sensitivity independent of the plasma adiponectin level
}

\author{
Y.-C. Hwang • I.-K. Jeong • K.-J. Ahn • H.-Y. Chung
}

Received: 14 March 2011 / Accepted: 9 May 2011 /Published online: 9 June 2011

(C) The Author(s) 2011. This article is published with open access at Springerlink.com

\begin{abstract}
Summary In agreement with the results of animal studies, the plasma osteocalcin level is positively associated with improved glucose tolerance and insulin secretion and sensitivity. In addition, the plasma osteocalcin level is inversely associated with the development of diabetes; however, the plasma adiponectin level may not be involved in osteocalcin-mediated energy metabolism in humans.

Introduction Recent animal studies have suggested crosstalk between bone and energy metabolism through osteocalcin. The aims of this study were to determine whether or not osteocalcin is associated with the improved glucose tolerance and insulin secretion and sensitivity, and whether or not the association is dependent on the plasma adiponectin level in humans.

Methods Four hundred twenty-five subjects, 19-82 years of age (mean age, 53 years), were enrolled. An oral glucose tolerance test (OGTT) and OGTT-based methods that were validated against the euglycemic clamp were determined. Total osteocalcin, leptin, and total adiponectin levels were measured.

Results The plasma levels of total osteocalcin were significantly different between the normal glucose tolerance, pre-diabetes, and diabetes groups. The glucose levels and homeostasis model assessment insulin resis-
\end{abstract}

Y.-C. Hwang • I.-K. Jeong • K.-J. Ahn • H.-Y. Chung $(\bowtie)$

Division of Endocrinology and Metabolism,

Department of Medicine, Kyung Hee University Hospital

at Gangdong, Kyung Hee University School of Medicine,

\#149, Sangil-dong, Kangdong-ku,

Seoul 134-727, South Korea

e-mail: hychung@khnmc.or.kr tance values varied inversely with the osteocalcin tertiles, and OGTT-based insulin secretion (HOMA-B\%, disposition index) and insulin sensitivity indices (Stumvoll's and OGIS indices) were increased with the tertiles. Although the plasma adiponectin level was positively correlated with the osteocalcin level, no changes in the association were noted between the plasma osteocalcin level and the glucose tolerance or insulin secretion and sensitivity indices after adjustment for the plasma adiponectin level. Based on multiple logistic regression analysis, the plasma osteocalcin level was inversely associated with the development of type 2 diabetes mellitus independent of age, gender, body mass index, and fasting plasma glucose and plasma adiponectin levels.

Conclusions Circulating osteocalcin level is associated with improved glucose tolerance and insulin secretion and sensitivity independent of the plasma adiponectin level in humans.

Keywords Adiponectin · Insulin resistance · Insulin secretion · Osteocalcin
Abbreviations
AUC Area under the curve
BMI Body mass index
FPG Fasting plasma glucose
FPI Fasting plasma insulin
HOMA Homeostasis model assessment
IFG Impaired fasting glucose
IGT Impaired glucose tolerance
OGIS Oral glucose insulin sensitivity
OGTT Oral glucose tolerance test
NGT Normal glucose tolerance
T2DM Type 2 diabetes mellitus 


\section{Introduction}

Recently, Lee et al. [1] have described a novel function of the skeleton on energy metabolism. Specially, they demonstrated that the osteoblast-specific protein, osteocalcin, is involved in glucose metabolism by increasing insulin secretion and cell proliferation in pancreatic $\beta$-cells and improving insulin sensitivity by upregulating the expression of an insulinsensitizing adipokine (the adiponectin gene) in adipocytes.

Subsequent human studies, including our own work, have confirmed the previous report [2-10]. Collectively, these human studies have shown that the serum osteocalcin concentration is negatively associated with the plasma glucose level and body fat mass [3, 5-7] and positively associated with insulin secretion [4, 8], lower insulin resistance [5-9], and serum adiponectin concentration [3,9]. In addition, Kanazawa et al. [3] showed that the serum osteocalcin level is negatively associated with the brachial-ankle pulse wave velocity and carotid intima-media thickness and suggested that osteocalcin might, thus, be linked to atherosclerosis.

To date, homeostasis model assessment (HOMA) values have mainly been used to assess $\beta$-cell function and insulin sensitivity and the involvement of osteocalcin on glucose metabolism. However, the HOMA $\beta$-cell function index (HOMA-B\%) is proportional to the fasting insulin level and is expected to be inversely related to insulin sensitivity in subjects with normal glucose tolerance (NGT), and thus, adjustment for insulin sensitivity is necessary [11]. Also, the agreement between homeostasis model assessment insulin resistance (HOMA-IR), an indicator of insulin resistance, and clampmeasured insulin sensitivity is controversial, ranging from very good to nonexistent [12]. Therefore, it is necessary to determine the association between osteocalcin and insulin secretion and insulin sensitivity with more valid methods. In addition, it remains uncertain whether or not the insulinsensitizing and glucose-lowering effects of osteocalcin are truly mediated by upregulation of the adiponectin gene in humans. Therefore, we performed a study to determine whether or not osteocalcin is positively correlated with insulin secretion and insulin sensitivity using oral glucose tolerance test (OGTT)based methods that have been validated against the clamp. In addition, we determined whether or not osteocalcin is inversely associated with the development of type 2 diabetes mellitus (T2DM) after adjusting for other diabetes risk factors and the plasma adiponectin level in humans.

\section{Methods}

\section{Subjects}

We recruited study subjects from among those who attended the Kyung Hee University Hospital at Gangdong between December 2006 and July 2009 for the diagnosis, evaluation, or treatment of diabetes. During this period, 1,785 subjects (942 males and 843 females) underwent a 75-g OGTT, and after acquiring informed consent, plasma samples were obtained and stored at $-70 \mathrm{C}$ for future studies involving cardiovascular disorders and diabetes. The exclusion criteria applied were as follows: (1) history of metabolic bone diseases, such as hyperparathyroidism; (2) uncontrolled liver or thyroid diseases; (3) acute illnesses, such as infection, surgery, and hospital admission for a medical condition other than diabetes; (4) recent history of a fracture ( $<6$ months); and (5) medications known to affect bone or glucose metabolism, such as glucocorticoids or bisphosphonates.

In this study, diabetes mellitus was defined by the presence of one of the following: (1) fasting glucose levels at $\geq 126 \mathrm{mg} / \mathrm{dl}(\geq 7.0 \mathrm{mmol} / \mathrm{l})$ or (2) 2 -h post-load glucose levels at $\geq 200 \mathrm{mg} / \mathrm{dl}(\geq 11.1 \mathrm{mmol} / \mathrm{l})$. To eliminate the effects of drugs on insulin secretion and sensitivity driven by OGTT, we limited our study subjects to those who had never been treated with oral glucose-lowering agents to eliminate the effects on glucose tolerance, and insulin secretion and sensitivity measured by OGTT. Finally, 425 subjects, 19-82 years of age (mean age, 53.0 12.0 years), were enrolled in this study. According to the OGTT results, subjects were diagnosed as follows: NGT $(n=23)$; prediabetes $(n=150)$, which included subjects with impaired fasting glucose (IFG), impaired glucose tolerance (IGT), and both IFG and IGT; and diabetes $(n=252)$. The study was approved by the Ethics Committee and the Institutional Review Board of Kyung Hee University Hospital and complied with the Declaration of Helsinki.

\section{Biochemical measurements}

After an overnight fast ( $8 \sim 12 \mathrm{~h})$, a 75 -g OGTT was begun between 0800 and 0900 hours according to standardized clinical procedures. In brief, after a cannula was inserted in an antecubital vein for blood sampling, basal blood samples were drawn $(0 \mathrm{~min})$, then $75 \mathrm{~g}$ of glucose (Diasol ${ }^{\circledR}$, dissolved in $300 \mathrm{ml}$ of water) was consumed within 5 min. Plasma glucose and insulin levels were then determined 30, 60, 90, and $120 \mathrm{~min}$ after the glucose had been administered. In addition, the plasma C-peptide levels were measured at time 0 and $30 \mathrm{~min}$.

To estimate insulin sensitivity, HOMA was used based on fasting plasma glucose (FPG) and fasting plasma insulin (FPI) concentrations. Insulin resistance was estimated using HOMA-IR, which was defined as follows: (FPI $(\mu \mathrm{U} / \mathrm{mL}) \times \mathrm{FPG}(\mathrm{mmol} / \mathrm{L})) / 22.5$. In addition, we estimated insulin sensitivity in the subjects using the three most extensively validated OGTT insulin sensitivity indices against the euglycemic clamp tech- 
nique in a relatively large numbers of subjects (ISIcomp [13], MCRest [14], and OGIS [15]). To estimate $\beta$-cell function, HOMA-B $\%$ was calculated as follows: $(20 \times$ $\mathrm{FPI} /(\mathrm{FPG}-3.5)$. The insulinogenic index was defined as the ratio of insulin change to plasma glucose change 30 min after a 75 -g oral glucose load ( $\Delta$ insulin, 0 $30 \mathrm{~min} / \Delta$ plasma glucose, $0-30 \mathrm{~min}$ ) and was used to estimate early phase insulin secretion. In addition, the area under the curve (AUC) of glucose or insulin levels during the OGTT was calculated by the trapezoidal rule, and the ratio of the total AUC insulin to the total AUC glucose (total AUC insulin/glucose) was used to measure the summation of the total insulin secretory capacity [16]. The disposition index was defined as the product of the insulinogenic index and Matsuda's index and was used for estimating the insulin secretory capacity adjusted for insulin resistance.

The plasma glucose levels were determined using the hexokinase method in an autoanalyzer (Hitachi, Tokyo, Japan), which had a CV of $1.7 \%$. The plasma insulin (Biosource, Nivelles, Belgium) and C-peptide levels (Immunotech, Czech Republic) were determined using immunoradiometric assays with intra- and inter-assay CVs of $1.6-2.2 \%$ and $6.1-6.5 \%$ and $2.3-3.0 \%$ and $3.5-$ $5.1 \%$, respectively. The plasma total osteocalcin was measured with an IRMA method using an Osteo-RIACT kit from Cis Bio International (Saclay, France), which had intra- and inter-assay CVs of $1.2-2.8 \%$ and $3.6-$ $5.2 \%$, respectively. Total plasma adiponectin and leptin levels were measured by ELISA kits (R\&D Systems, Minneapolis, MN, USA), as recommended by the manufacturer.

\section{Statistical methods}

All data are presented as the means \pm SDs or proportions, except for skewed variables, which were presented as the median (interquartile range, 25-75\%). Because the distributions of fasting and 2-h plasma insulin levels, AUC insulin, AUC insulin/glucose, HbA1c level, HOMA values, insulinogenic index, disposition index, adiponectin level, and leptin level were skewed as assessed by the Kolmogorov-Smirnov test, the natural logarithmic transformation was applied in the statistical analysis. In the interests of simplicity, nontransformed median values are presented in the tables and text. One-way ANOVA, followed by Turkey's post hoc test, was used to compare the means between the tertiles of osteocalcin levels. Pearson correlation coefficients were calculated to evaluate the associations between osteocalcin and age, body mass index (BMI), and metabolic parameters (glucose, insulin, and insulin secretory and insulin sensitivity indices). Multiple linear regression analysis was used to determine the associations between plasma osteocalcin and glucose tolerance, and insulin secretory or sensitivity indices after adjusting for age, gender, BMI, and other adipokines, including adiponectin and leptin levels. To identify whether or not plasma total osteocalcin was independently associated with the development of T2DM, we performed a multivariate logistic regression analysis with backward variable selection. Analysis was performed using SPSS (version 13.0; SPSS, Inc. Chicago, IL, USA), and $p$ values of $<0.05$ were considered significant.

\section{Results}

We divided the study subjects according to glucose tolerance status, and compared the plasma total osteocalcin levels. The plasma osteocalcin levels were significantly different between the groups $(p<0.001)$; however, no difference was noted in the osteocalcin levels between the NGT $(18.4 \pm 9.0 \mathrm{ng} / \mathrm{ml})$ and pre-diabetes groups $(19.1 \pm$

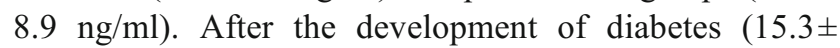
$6.8 \mathrm{ng} / \mathrm{ml}$ ), the plasma osteocalcin levels were decreased compared with the pre-diabetes group (Fig. 1). Next, we divided the subjects into tertiles (lower, middle, and upper) by plasma osteocalcin levels; the glucose and HbAlc levels varied inversely with the osteocalcin tertiles, and the insulin secretory capacity, including the AUC insulin/glucose, HOMA-B\%, insulinogenic index, and disposition index and insulin sensitivity index (Matsuda's, Stumvoll's, and OGIS indices), increased with the osteocalcin tertiles. In addition, the plasma adiponectin levels were increased with the osteocalcin tertiles; however, no difference was noted in the plasma leptin levels with the osteocalcin tertiles (Table 1). To determine whether or not plasma osteocalcin level is independently associated with improved glucose tolerance and insulin sensitivity and secretory capacity, multiple linear regression analyses were performed. The plasma osteocalcin level was inversely associated with FPG and AUC glucose levels and positively associated with the disposition index

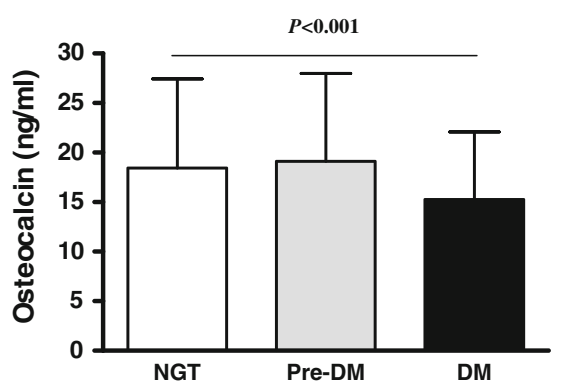

Fig. 1 Osteocalcin levels (means \pm SDs) by glucose tolerance status. $N G T$ normal glucose tolerance, Pre- $D M$ pre-diabetes, $D M$ diabetes. To convert osteocalcin levels to nanomoles per liter, multiply by 0.171 
Table 1 Insulin secretion and sensitivity by osteocalcin tertiles

\begin{tabular}{|c|c|c|c|c|}
\hline Variable & $\begin{array}{l}\text { Osteocalcin lower tertile } \\
(n=141)\end{array}$ & $\begin{array}{l}\text { Osteocalcin middle tertile } \\
(n=143)\end{array}$ & $\begin{array}{l}\text { Osteocalcin upper tertile } \\
(n=141)\end{array}$ & $p$ \\
\hline Osteocalcin (ng/ml, range) & $9.1 \pm 3.2(1.1-13.0)$ & $15.7 \pm 1.7(13.1-18.9)$ & $25.3 \pm 6.7(19.0-70.7)$ & $<0.001$ \\
\hline Age (year) & $53.2 \pm 13.1$ & $52.0 \pm 11.9$ & $54.0 \pm 10.9$ & NS \\
\hline Gender (male/female) & $74 / 67$ & $89 / 54$ & $60 / 81$ & NS \\
\hline BMI $\left(\mathrm{kg} / \mathrm{m}^{2}\right)$ & $25.3 \pm 3.5$ & $25.5 \pm 3.8$ & $25.5 \pm 3.8$ & NS \\
\hline Glucose $\left(0^{\prime}\right)(\mathrm{mg} / \mathrm{dl})$ & $155.0 \pm 66.7$ & $126.1 \pm 30.6$ & $118.9 \pm 28.8$ & $<0.001$ \\
\hline Insulin $\left(0^{\prime}\right)(\mu \mathrm{IU} / \mathrm{ml})$ & $10.1(7.2-14.5)$ & $10.7(8.4-14.2)$ & $9.9(7.4-12.9)$ & 0.046 \\
\hline HbAlc (\%) & $7.7 \pm 2.4$ & $6.6 \pm 1.3$ & $6.4 \pm 1.3$ & $<0.001$ \\
\hline AUC glucose $\left(0-120^{\prime}\right)$ & $28.2 \pm 10.7$ & $24.1 \pm 6.8$ & $22.8 \pm 6.9$ & $<0.001$ \\
\hline AUC insulin $\left(0-120^{\prime}\right)$ & $323.2(204.9-573.6)$ & $438.2(280.6-693.0)$ & $400.5(263.7-662.9)$ & $<0.001$ \\
\hline AUC insulin/glucose $\left(0-120^{\prime}\right)$ & $13.5(7.0-26.0)$ & $18.4(11.6-34.9)$ & 19.7 (11.4-31.9) & $<0.001$ \\
\hline HOMA-IR & $3.44(2.45-5.21)$ & $3.47(2.52-4.26)$ & $2.82(2.05-3.87)$ & 0.002 \\
\hline HOMA-B \% & $58.6(32.0-91.7)$ & $74.2(49.0-104.8)$ & $75.5(54.6-97.5)$ & $<0.001$ \\
\hline Insulinogenic index & $0.18(0.08-0.44)$ & $0.29(0.15-0.58)$ & $0.32(0.14-0.57)$ & $<0.001$ \\
\hline Matsuda's index & $4.12 \pm 2.01$ & $3.85 \pm 1.81$ & $4.53 \pm 2.22$ & 0.018 \\
\hline Disposition index & $0.63(0.27-1.53)$ & $1.04(0.50-1.86)$ & $1.09(0.60-2.30)$ & $<0.001$ \\
\hline Stumvoll's index & $6.40 \pm 2.24$ & $6.57 \pm 2.72$ & $7.10 \pm 2.22$ & 0.040 \\
\hline OGIS index & $324.0 \pm 76.9$ & $350.3 \pm 57.3$ & $369.7 \pm 57.4$ & $<0.001$ \\
\hline Plasma adiponectin level $(\mu \mathrm{g} / \mathrm{ml})$ & $2.20(1.44-2.93)$ & $1.80(1.35-3.20)$ & $2.43(1.68-3.83)$ & $<0.001$ \\
\hline Plasma leptin level $(\mu \mathrm{g} / \mathrm{l})$ & $5.44(2.28-13.89)$ & $4.82(2.66-8.37)$ & $4.57(1.72-14.80)$ & NS \\
\hline
\end{tabular}

Data are presented as the means \pm SDs or median (interquartile range, 25-75\%), except as otherwise indicated. To convert glucose levels to milimoles per liter, multiply by 0.0555 . To convert insulin levels to picomoles per liter, multiply by 6.945

$B M I$ body mass index, AUC area under the curve, HOMA homeostasis model assessment, ND not determined, $N S$ not significant

and Stumvoll's and OGIS indices after adjusting for age, gender, BMI, and other adipokines including adiponectin and leptin levels (Table 2). To investigate the independent association between the osteocalcin level and diabetes, a multiple logistic regression analysis was performed. The analysis included age, gender, BMI, fasting plasma glucose level, and plasma adiponectin, leptin, and osteocalcin levels. Our results indicated that age and the fasting plasma glucose level appeared to be independently associated with the development of diabetes; the plasma osteocalcin level was inversely associated with the development of diabetes (OR, 0.955; 95\% CI, 0.9190.994, $p=0.023$; Table 3).

\section{Discussion}

In the present study, the plasma levels of osteocalcin were inversely correlated with fasting and 2-h post-load plasma glucose levels and AUC glucose during an OGTT. In addition, the osteocalcin level was positively associated with the parameters which were estimated by the OGTT, reflecting $\beta$-cell function and insulin sensitivity. Until now, the associations between osteocalcin and insulin secretion and sensitivity were primarily measured by HOMA values; however, the model predicts the fasting steady-state glucose and insulin concentrations for a wide range of possible combinations of insulin resistance and $\beta$-cell function, and it is difficult to determine the true dynamic function of $\beta$ cell insulin secretion. In addition, in subjects with severely impaired $\beta$-cell function, HOMA-IR did not represent appropriate insulin resistance status [17], and therefore the agreement between HOMA-IR and clamp-measured insulin sensitivity remains controversial [12]. The current study was unique and powered because we determined the association between plasma osteocalcin levels and insulin sensitivity with OGTT-driven dynamic methods that have been extensively validated against euglycemic clamp methods, and determined the $\beta$-cell function with diverse parameters, including the HOMA-B\%, insulinogenic index, AUC insulin/glucose, and disposition index.

According to the original observation by Lee et al. [1], osteocalcin regulates insulin sensitivity, at least in part, through adiponectin gene expression. In the current study, the plasma adiponectin levels were significantly different across the osteocalcin tertiles $(p<0.001)$ and were positively correlated with the indices representing insulin sensitivity, including Matsuda's, Stumvoll's, and OGIS indices (data not shown, all $p<0.01$ ). In multiple linear regression analyses, however, the plasma osteocalcin levels were still signifi- 
Table 2 Multiple linear regression analysis for glucose tolerance and insulin secretion and sensitivity indices

\begin{tabular}{|c|c|c|c|c|c|c|}
\hline Variable & FPG & AUC glucose $\left(0-120^{\prime}\right)$ & Disposition index & Matsuda's index & Stumvoll's index & OGIS index \\
\hline Age & -0.048 & 0.030 & $-0.170 * * *$ & $-0.110^{*}$ & $-0.104 *$ & -0.066 \\
\hline BMI & -0.029 & 0.016 & -0.077 & $-0.325 * * *$ & $-0.526^{* * *}$ & $-0.142 * *$ \\
\hline Adiponectin & -0.092 & $-0.131 * *$ & $0.134 * *$ & 0.059 & 0.048 & $0.141 * *$ \\
\hline Leptin & -0.081 & -0.098 & $0.127^{*}$ & $-0.182 * * *$ & -0.047 & 0.029 \\
\hline Osteocalcin & $-0.269 * * *$ & $-0.255 * * *$ & $0.142 * *$ & 0.064 & $0.141 * *$ & $0.240 * * *$ \\
\hline
\end{tabular}

Standard $\beta$ values from multiple linear regression analysis

$B M I$ body mass index

${ }^{*} p<0.05 ;{ }^{* *} p<0.01 ; * * * p<0.001$

cantly associated with improved glucose tolerance and insulin secretion and sensitivity indices even after controlling for the adiponectin levels. Therefore, adiponectin did not mediate the association between the osteocalcin level and glucose tolerance and insulin secretion and sensitivity in humans. In addition, we investigated whether or not the plasma osteocalcin level is inversely associated with the development of T2DM. The results indicated that the plasma osteocalcin level is inversely associated with the development of T2DM independent of well-established risk factors for diabetes, such as age, gender, BMI, and baseline fasting plasma glucose level and circulating adipokines including plasma adiponectin and leptin levels. These results suggest that osteocalcinmediated increased insulin sensitivity may not involve adiponectin gene upregulation in humans but may involve other mechanisms. This is the first report to demonstrate an independent association, especially independent of plasma adiponectin levels, between plasma osteocalcin levels and improved glucose tolerance and insulin secretion and sensitivity. In contrast with our results, Shea et al. [9] reported that the strength of the association between total osteocalcin and carboxylated osteocalcin with HOMA-IR is somewhat attenuated after adiponectin is accounted for; therefore, they concluded that the association between total osteocalcin and carboxylated osteocalcin with HOMA-IR may depend partially on adiponectin. Although we could not explain the discrepancy between the studies, the different

Table 3 Multiple logistic regression analysis for diabetes

\begin{tabular}{lcr}
\hline Variable & $\begin{array}{l}\text { OR per 1-SD increase in } \\
\text { variable }(95 \% \text { CI })\end{array}$ & \multicolumn{1}{l}{$p$} \\
\hline Age & $1.577(1.152-2.160)$ & 0.005 \\
Fasting plasma glucose & $471.399(120.817-1,839.284)$ & $<0.001$ \\
Total osteocalcin & $0.726(0.533-0.988)$ & 0.042 \\
\hline
\end{tabular}

Age, gender, body mass index, fasting plasma glucose, plasma adiponectin, leptin, and osteocalcin levels were included as dependent variables levels of insulin resistance between the study subjects and different measurements assessing insulin sensitivity may be casual.

In the current study, no difference in the osteocalcin level was noted between the NGT and pre-diabetes groups, and the level of the pre-diabetes group was somewhat higher compared with the NGT group, although it did not reach statistical significance. Therefore, it is not until diabetes develops that plasma osteocalcin levels are decreased. As a plausible explanation for this finding, it is possible that osteoblasts may secrete more osteocalcin to overcome a given amount of insulin resistance, and more insulin is initially secreted in pancreatic $\beta$-cells (prediabetes state). However, as insulin resistance becomes more severe, the osteoblast fails to secrete sufficient osteocalcin, insulin secretion is decreased, and diabetes finally develops. In partial agreement with our speculation, Winhofer et al. [10] reported that women with gestational diabetes have higher osteocalcin levels compared with women with NGT during pregnancy while no difference was observed between the two groups 12 weeks postpartum, and therefore, they hypothesized that osteocalcin can enhance insulin secretion in insulin-resistant states.

This study had several limitations. First, this study was based on a cross-sectional analysis, and thus, we do not know whether or not our findings are merely correlations or if osteocalcin has direct glucose-lowering effects in human subjects, as in animal- and cell-based studies. Second, we did not differentiate plasma osteocalcin with respect to the gamma-carboxylation status, and only measured the total form of osteocalcin, instead of directly measuring carboxylated and uncarboxylated osteocalcin. Therefore, we do not know the differential mechanism of both types of osteocalcin to regulate insulin secretion and insulin sensitivity. Third, it is known that the levels of bone turnover markers, including plasma osteocalcin, are different according to age, gender, and race or ethnicity [18]. In this study, although we adjusted for age and gender, we could not entirely exclude the effects of age and gender on the associations between plasma osteocalcin levels and glucose 
metabolism. Lastly, it has been suggested that bone resorption at low $\mathrm{pH}$ is necessary to decarboxylate osteocalcin, and thus, osteoclasts determine the carboxylation status and function of osteocalcin in mice [19] and possibly in humans [20]. Therefore, the additional measurement of bone resorption markers may further clarify the potential association between bone resorption, osteocalcin, and glucose homeostasis in humans.

In conclusion, an elevated plasma osteocalcin level was associated with improved glucose tolerance and may be associated with increased $\beta$-cell function and insulin sensitivity. However, it appears that the glucose-lowering and insulin-sensitizing effect of osteocalcin is not mediated by an increment in the plasma adiponectin level in humans.

Acknowledgment This research was supported by the Program of Kyung Hee University for the Young Researcher of Medical Science (KHU-20091457).

\section{Conflicts of interest None.}

Open Access This article is distributed under the terms of the Creative Commons Attribution Noncommercial License which permits any noncommercial use, distribution, and reproduction in any medium, provided the original author(s) and source are credited.

\section{References}

1. Lee NK, Sowa H, Hinoi E et al (2007) Endocrine regulation of energy metabolism by the skeleton. Cell 130:456-469

2. Im JA, Yu BP, Jeon JY, Kim SH (2008) Relationship between osteocalcin and glucose metabolism in postmenopausal women. Clin Chim Acta 396:66-69

3. Kanazawa I, Yamaguchi T, Yamamoto M et al (2009) Serum osteocalcin level is associated with glucose metabolism and atherosclerosis parameters in type 2 diabetes mellitus. J Clin Endocrinol Metab 94:45-49

4. Zhou M, Ma X, Li H et al (2009) Serum osteocalcin concentrations in relation to glucose and lipid metabolism in Chinese individuals. Eur J Endocrinol 161:723-729

5. Fernandez-Real JM, Izquierdo M, Ortega $F$ et al (2009) The relationship of serum osteocalcin concentration to insulin secre- tion, sensitivity, and disposal with hypocaloric diet and resistance training. J Clin Endocrinol Metab 94:237-2459

6. Kindblom JM, Ohlsson C, Ljunggren O et al (2008) Plasma osteocalcin is inversely related to fat mass and plasma glucose in elderly Swedish men. J Bone Miner Res 24:785-791

7. Pittas AG, Harris SS, Eliades M, Stark P, Dawson-Hughes B (2009) Association between serum osteocalcin and markers of metabolic phenotype. J Clin Endocrinol Metab 94:827-832

8. Hwang YC, Jeong IK, Ahn KJ, Chung HY (2009) The uncarboxylated form of osteocalcin is associated with improved glucose tolerance and enhanced beta-cell function in middle-aged male subjects. Diab Metab Res Rev 25:768-772

9. Shea MK, Gundberg CM, Meigs JB et al (2009) Gammacarboxylation of osteocalcin and insulin resistance in older men and women. Am J Clin Nutr 90:1230-1235

10. Winhofer Y, Handisurya A, Tura A et al (2010) Osteocalcin is related to enhanced insulin secretion in gestational diabetes. Diabetes Care 33:139-143

11. Mari A, Ahrén B, Pacini G (2005) Assessment of insulin secretion in relation to insulin resistance. Curr Opin Clin Nutr Metab Care 8:529-533

12. Pacini G, Mari A (2003) Methods for clinical assessment of insulin sensitivity and beta-cell function. Best Pract Res Clin Endocrinol Metab 17:305-322

13. Matsuda M, DeFronzo RA (1999) Insulin sensitivity indices obtained from oral glucose tolerance testing: comparison with the euglycemic insulin clamp. Diabetes Care 22:1462-1470

14. Stumvoll M, Mitrakou A, Pimenta W et al (2000) Use of the oral glucose tolerance test to assess insulin release and insulin sensitivity. Diabetes Care 23:295-301

15. Mari A, Pacini G, Murphy E, Ludvik B, Nolan JJ (2001) A model-based method for assessing insulin sensitivity from the oral glucose tolerance test. Diabetes Care 24:539-548

16. Ahren B, Pacini G (2004) Importance of quantifying insulin secretion in relation to insulin sensitivity to accurately assess $\beta$ cell function in clinical studies. Eur J Endocrinol 150:97-104

17. Muniyappa R, Lee S, Chen H, Quon MJ (2008) Current approaches for assessing insulin sensitivity and resistance in vivo: advantages, limitations, and appropriate usage. Am J Physiol Endocrinol Metab 294:E15-E26

18. Gundberg CM, Looker AC, Nieman SD, Calvo MS (2002) Patterns of osteocalcin and bone specific alkaline phosphatase by age, gender, and race or ethnicity. Bone 31:703-708

19. Ferron M, Wei J, Yoshizawa T et al (2010) Insulin signaling in osteoblasts integrates bone remodeling and energy metabolism. Cell 142:296-308

20. Aonuma H, Miyakoshi N, Hongo M, Kasukawa Y, Shimada Y (2009) Low serum levels of undercarboxylated osteocalcin in postmenopausal osteoporotic women receiving an inhibitor of bone resorption. Tohoku J Exp Med 218:201-205 